\title{
Auditory apparent motion in the free field: The effects of stimulus duration and separation
}

\author{
THOMAS Z. STRYBEL and APRIL M. WITTY \\ California State University, Long Beach, California \\ and \\ DAVID R. PERROTT \\ California State University, Los Angeles, California
}

\begin{abstract}
The effects of stimulus duration and spatial separation on the illusion of apparent motion in the auditory modality were examined. Two narrow-band noise sources (40 dB, A-weighted) were presented through speakers separated in space by $2.5^{\circ}, 5^{\circ}$, or $10^{\circ}$, centered about the subject's midline. The duration of each stimulus was 5,10 , or $50 \mathrm{msec}$. On each trial, the sound pair was temporally separated by 1 of 10 interstimulus onset intervals (ISOIs): $0,2,4,6,8,10,15,20$, 50 , or 70 msec. Five subjects were tested in nine trial blocks; each block represented a particular spatial-separation-duration combination. Within a trial block, each ISOI was presented 30 times each, in random order. Subjects were instructed to listen to the stimulus sequence and classify their perception of the sound into one of five categories: single sound, simultaneous sounds, continuous motion, broken motion, or successive sounds. Each subject was also required to identify the location of the first-occurring stimulus (left or right). The percentage of continuous-motion responses was significantly affected by the ISOI $[F(9,36)=5.67, p<.001]$, the duration $\times$ ISOI interaction $[F(18,72)=3.54, p<.0001]$, and the separation $\times$ duration $\times$ ISOI interaction $[F(36,144)=1.51, p<.05]$. The results indicate that a minimum duration is required for the perception of auditory apparent motion. Little or no motion was reported at durations of 10 msec or less. At a duration of $50 \mathrm{msec}$, motion was reported most often for ISOIs of 20-50 msec. The effect of separation appeared to be limited to durations and ISOIs during which little motion was perceived
\end{abstract}

The illusion of apparent motion is a general sensory phenomenon, because it occurs in the visual, auditory, and tactual modalities. Visual apparent motion has been studied extensively, and it is probably the dominant means of investigating visual motion perception. On the other hand, the illusion of auditory apparent motion has received only minimal attention in the literature. In such work, the auditory illusion has usually been compared with the visual illusion.

The search for similarities between these illusions has focused on the applicability of Korte's laws (Kolers, 1972; Korte, 1915) to the auditory illusion. Korte determined the relationship between the duration of the stimulus, the interstimulus interval (or interstimulus onset interval, ISOI), the spatial separation between the stimuli, and the intensity of the stimuli. Two of these laws (the second and third) have been studied in the auditory modality.

Korte's (1915) second law describes the relationship between the ISOI and duration. This law states that for visual apparent motion to be maintained, when the duration of the stimuli is increased, the ISOI must also increase, but

Correspondence should be addressed to T. Z. Strybel, Department of Psychology, California State University, Long Beach, CA 90840. the ISOI increases at a slower rate. This law was verified in the visual modality by Neuhaus (1930), and it is described in Kolers (1972). For a duration of $10 \mathrm{msec}$, the smallest ISOI that produced apparent motion was $70 \mathrm{msec}$. For a 90 -msec duration, this ISOI increased to $130 \mathrm{msec}$. Therefore, increasing the duration by $900 \%$ requires that the ISOI be increased by only $180 \%$, in order for the perception of visual apparent motion to be maintained.

Briggs and Perrott (1972) tested the applicability of this law to auditory apparent motion under dichotic listening conditions. Stimulus durations between 10 and $300 \mathrm{msec}$ were tested. Continuous motion was produced with an optimum ISOI of roughly $20 \mathrm{msec}$ when the duration was $10 \mathrm{msec}$, and $70 \mathrm{msec}$ when the duration was $300 \mathrm{msec}$. The relationship between duration and ISOI was the same as that obtained by Korte (1915), but two differences between the auditory and visual illusion were observed. First, the slope of the function relating ISOI to duration is lower in audition. A 3,000\% increase in duration required only a $400 \%$ increase in ISOI-much less than the increase reported by Neuhaus (1930) for visual apparent motion. Second, auditory apparent motion is heard at lower overall values of ISOI (e.g., at a 10-msec duration, 
70 -msec ISOI in vision vs. $20 \mathrm{msec}$ in audition). However, Strybel, Manligas, Chan, and Perrott (1990) determined that auditory and visual apparent motion can be perceived at the same ISOI when the separation of the visual stimuli is less than $2^{\circ}$.

Korte's (1915) third law describes the relationship between the ISOI and the spatial separation required to produce visual apparent motion. This law states that as the spatial separation increases, the ISOI must also increase, in order to produce apparent motion. This law was recently tested in the auditory and visual modalities. Strybel et al. (1990) showed that the ISOI range that produces auditory apparent motion was constant at $20-45 \mathrm{msec}$ for separations between $6^{\circ}$ and $160^{\circ}$. The ISOI range that produced visual apparent motion depended on the spatial separation. For separations between $.5^{\circ}$ and $2^{\circ}$, continuous motion was obtained for ISOIs between 20 and $45 \mathrm{msec}$. For separations between $4^{\circ}$ and $40^{\circ}$, this range increased to $45-130 \mathrm{msec}$. At separations greater than $40^{\circ}$, little motion was perceived. Strybel et al. concluded that spatial separation has no effect on auditory apparent motion, but that spatial separation determines the range of ISOIs that produce visual apparent motion. Furthermore, there is an upper limit on the spatial separation at which visual apparent motion can be perceived, but no upper limit on spatial separation in the auditory modality.

Research in which visual and auditory apparent motion have been compared suggests that the auditory illusion is a more "robust" illusion. Whereas the visual illusion is affected by stimulus duration and separation, the auditory illusion is affected only by duration, and the magnitude of the effect is less in the auditory modality. Furthermore, the visual illusion is limited by the separation. No upper limit has been found for the auditory illusion. Additional research on the auditory illusion has yielded no effect of either the interaural correlation between the lag and lead signal or the rise/decay time of each signal (Perrott, 1974). Apparent motion is also heard under monaural as well as binaural listening conditions (Strybel, Manligas, \& Perrott, 1989). On the other hand, research on visual apparent motion has elucidated numerous other influences on visual apparent motion (for reviews, see Anstis, 1978; Kolers, 1972).

One could conclude, on the basis of the auditory apparent motion data, that auditory apparent motion is dependent primarily on the ISOI. Presumably, any two signals could produce the perception motion as long as they were properly timed. However, very little data is available on the auditory illusion, and it is possible that the relevant variables have not yet been discovered. The present investigation was undertaken for this reason. We determined whether conditions could be arranged so that no motion was experienced when two signals were presented at ISOIs that normally produce continuous motion. One of the stimulus factors tested here was suggested in the research of Briggs and Perrott (1972) on the effects of stimulus duration. In the $10-\mathrm{msec}$ duration condition, Briggs and Perrott reported that continuous motion was heard most often at an ISOI of $20 \mathrm{msec}$. However, at this ISOI, the mean percentage of continuous-motion reports was only $50 \%$. At longer durations, the magnitude of these peaks was $80 \%$ or higher. This observation suggests that a minimum signal duration may be required for the auditory illusion. A second factor examined in the present investigation was the separation between the stimuli. We determined whether a minimum separation is required for the perception of auditory apparent motion by testing a lower range of separations than has been used previously.

The purpose of the present investigation was, therefore, to further examine the relationship between duration, ISOI, and separation in the auditory modality under freefield listening conditions. We determined whether the illusion could be heard with very short pulses, by measuring the percentage of motion reports for both a lower range of durations $(5-50 \mathrm{msec})$ and a smaller range of ISOIs than have been used previously. In addition, we tested each duration over three values of separation between $2.5^{\circ}$ and $10^{\circ}$.

\section{METHOD}

\section{Subjects}

Five subjects were tested, including one of the authors, A. W. All of the subjects were female and had normal hearing. Three of the subjects had previous experience with auditory apparent motion experiments.

\section{Apparatus}

The testing was conducted in a $2.7 \times 3.7 \times 2.1 \mathrm{~m}$ nonanechoic, audiometric chamber. Each subject sat in a chair in the center of the room, with her head fixed by a chinrest. Two Realistic $5.1-\mathrm{cm}$ loudspeakers were mounted $1 \mathrm{~m}$ in front of the subject at an elevation corresponding to the ear level of the subject. The separation between the speakers could be adjusted from $2.5^{\circ}$ to $180^{\circ}$, centered about $0^{\circ}$ azimuth.

The stimulus, produced by an Amiga 1000 computer, consisted of a narrow band of noise centered at $4000 \mathrm{~Hz}$ with a $6-\mathrm{dB}$ /octave rolloff. (The limited bandwidth of the stimulus was due to the loudspeakers.) Each of the two sound channels of the computer was led through separate channels of an amplifier to the loudspeakers located in the chamber. The level of the noise was set at $40 \mathrm{~dB}$, A-weighted. The duration of each stimulus and the ISOI were controlled by the computer, and the subjects' responses were collected by a response box attached to the game port of the computer. This box contained five three-position, spring-loaded switches.

\section{Procedure}

Three stimulus durations $(5,10$, and $50 \mathrm{msec})$, and three spatial separations $\left(2.5^{\circ}, 5^{\circ}\right.$, and $\left.10^{\circ}\right)$ were used. Each duration-separation combination constituted a trial block, for a total of nine trial blocks per subject. The order of trial-block presentation was randomized for each subject. Within each trial block, 10 ISOIs $(0,2$, $4,6,8,10,15,20,50$, and $70 \mathrm{msec}$ ) were presented 30 times each, in random order, making a total of 300 presentations per trial block. At each ISOI, half of the 30 trials originated to the left of the subject, and half to the right.

Each subject was instructed to listen to the stimulus sequence and to classify her perception of the stimulus into one of five categories: Single sound (a single sound image was heard somewhere with no perceived movement); simultaneous sounds (two sounds were heard at the same time at two different locations with no movement be- 
tween them); continuous motion (a sound was heard at one side and then moved continuously to the other side); broken motion (a sound was heard to move from one side to the other, but the movement was interrupted or discontinuous); and succession (two sounds were heard in sequence, with no movement between them). In addition to classifying her perception of the sounds, the subject was also instructed to indicate the location of the first-occurring stimulus (left or right). On trials on which movement was heard, this could be determined by noting the direction of travel. On nonmoving trials, this response was determined by detecting the temporal order of the stimuli. The subject entered the stimulus category by pushing the appropriately labeled switch on the response box. The temporal order was entered by pushing the switch in the appropriate direction (left or right).

\section{RESULTS}

The percentage of responses in each category was obtained for every subject at each ISOI, duration and separation. Figures 1-3 show the mean percentage of responses in each category as a function of ISOI and duration. At all durations, the category used most often at ISOIs of $10 \mathrm{msec}$ or less was "single." For ISOIs greater than 8-10 msec, the response category used most often depended on the duration of the stimuli.

At the 5-msec duration condition, shown in Figure 1, the subjects' responses were divided among all of the categories for ISOIs greater than $8 \mathrm{msec}$. For example, at an ISOI of $15 \mathrm{msec}$, "continuous motion" was used most often, but the mean percentage of "continuous-motion" responses was only $36 \%$. The remaining $64 \%$ of the responses were divided among all of the other categories. At this duration, no one category seems to describe the subjects' perception of the stimulus sequence best. A similar pattern was obtained at the 10 -msec duration, shown in Figure 2. "Continuous motion" was again the response made most often at an ISOI of $15 \mathrm{msec}$, but this response was used only $46 \%$ of the time, on the average. At longer ISOIs, there does not seem to be a major category, with the exception of $70 \mathrm{msec}$, where the "succession"' category was used $52 \%$ of the time. However, at the 50 -msec duration, a different pattern emerges (see Figure 3). At an ISOI of 20 msec, "continuous motion" was reported

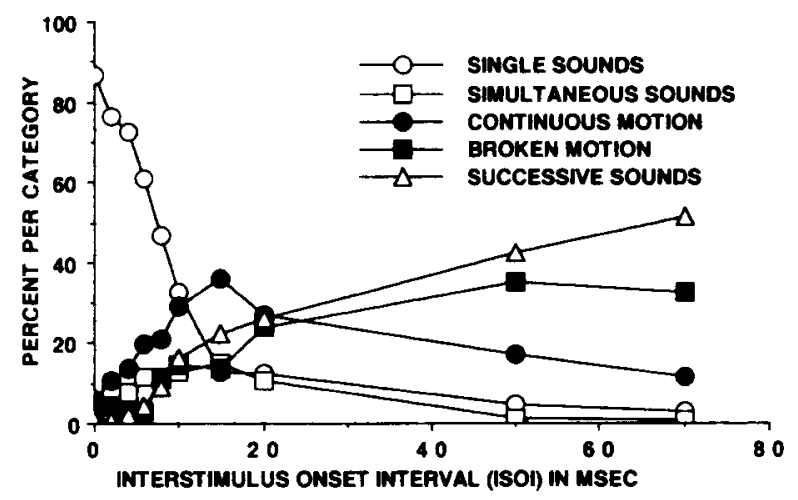

Figure 1. Mean percentage of responses in each category as a function of interstimulus onset interval for a signal duration of 5 msec.

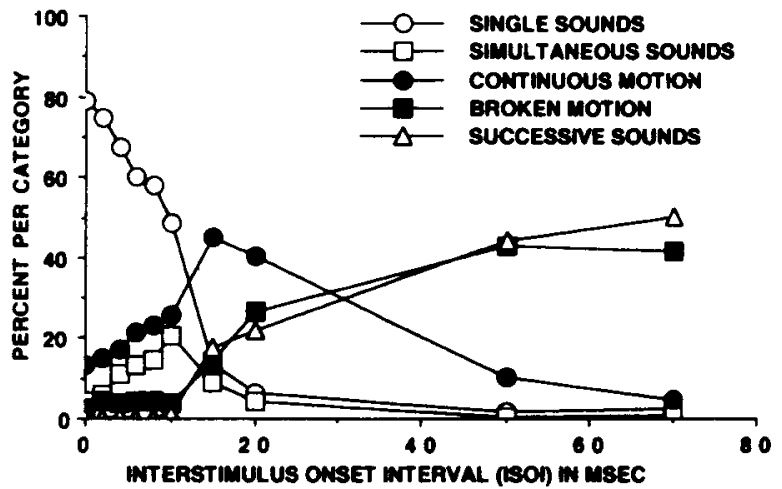

Figure 2. Mean percentage of responses in each category as a function of interstimulus onset interval for a signal duration of $10 \mathrm{msec}$.

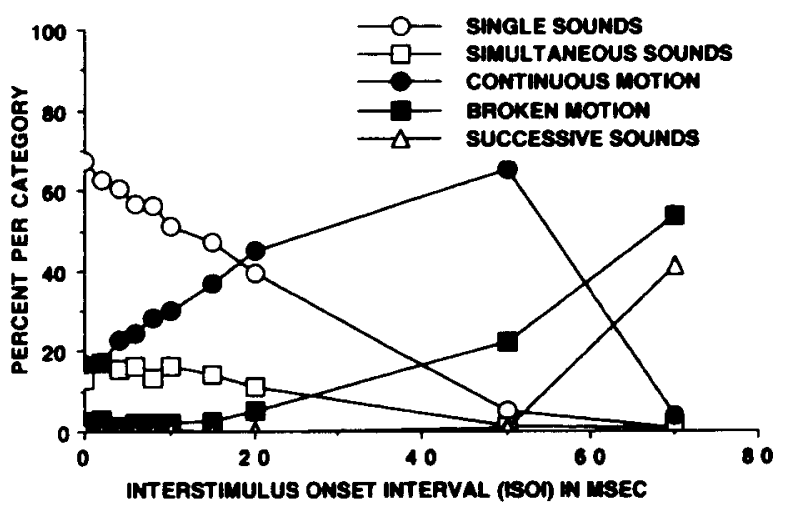

Figure 3. Mean percentage of responses in each category as a function of interstimulus onset interval for a sigmal duration of 50 msec.

$45 \%$ of the time, and at 50 msec this percentage increases to $65 \%$. At 70 msec, "broken motion" was reported 53\% of the time. Thus, the motion categories are clearly dominant in this ISOI range, and "continuous motion" is dominant for ISOIs between 20 and $50 \mathrm{msec}$.

Figures 1-3 suggest that the percentage of continuousmotion responses is affected by the interaction of ISOI and duration. A three-way repeated measures analysis of variance (ANOVA) confirmed this observation. This analysis was performed on the percentage of continuousmotion responses with ISOI, duration, and separation as factors. A significant main effect of ISOI was obtained $[F(9,36)=5.67, p<.001]$, as well as a significant interaction of ISOI with duration $[F(18,72)=3.54, p<$ $.0001]$, and a three-way interaction of ISOI with duration and separation $[F(36,144)=1.51, p<.05]$. The significant three-way interaction between ISOI, duration, and separation was limited to ISOIs and durations during which little motion was heard. Furthermore, this interaction accounted for only $2 \%$ of the total variability, while the duration $\times$ ISOI interaction accounted for $7 \%$. The main effect of ISOI accounted for $30 \%$ of the variance.

The effects of duration on the perception of continuous motion can be further verified by an examination of 
the individual subject data. Considerable variability between subjects was observed, but more subjects reported continuous motion at the longer durations. At a duration of $5 \mathrm{msec}$, only 1 subject reported continuous motion over $50 \%$ of the time at one or more ISOIs at all three separations. Of the remaining 4 subjects, 2 reported continuous motion at one separation, and 2 never exceeded $50 \%$ continuous-motion reports at any separation. At the 10msec duration, 2 subjects reported motion more than $50 \%$. of the time at all separations, 2 had $50 \%$ continuousmotion reports at two separations, and 1 failed to report continuous motion at any separation. At $50 \mathrm{msec}, 4$ out of 5 subjects reported continuous motion more than $50 \%$ of the time at all three separations. The remaining subject reported continuous motion at two separations.

Finally, an ANOVA was performed on the percentage of correct temporal order data. Overall performance on this task was very poor and rarely exceeded chance levels. Performance appears to be affected by the separation between the sources, but the differences are relatively small. Only the separation $\times$ ISOI interaction was significant $[F(18,72)=2.35, p<.01]$.

\section{DISCUSSION}

These results indicate that a minimum duration is necessary for the perception of auditory apparent motion. The mean percentage of continuous motion responses is very low at stimulus durations of 5 and $10 \mathrm{msec}$, and relatively few subjects reported continuous motion at these durations. On the other hand, at a duration of $50 \mathrm{msec}$, distinct continuous-motion distributions were obtained, and nearly all subjects reported continuous motion. It appears that a minimum duration of $10-50 \mathrm{msec}$ is required for the perception of auditory apparent motion, with the exact value dependent on the particular listener. Futhermore, this minimum duration requirement did not appear to be affected by the spatial separation between the sources.

The results obtained in the present experiment are consistent with previous experiments on auditory apparent motion. For example, in the present experiment, an ISOI of $15-20$ msec was required for continuous motion at stimulus durations of 5 and $10 \mathrm{msec}$, although motion was not often heard. The continuous-motion distribution obtained by Briggs and Perrott (1972) is very similar, for it has a shallow peak at $15 \mathrm{msec}$. In our 50 -msec duration condition, ISOIs of 20-50 msec produced continuous motion, which is consistent with previous work at this duration (e.g., Strybel et al., 1990; Strybel et al., 1989).

The effect of stimulus duration on the ISOIs that produce continuous motion was not affected by the separation between the sources. Although a significant threeway interaction between duration, separation, and ISOI was obtained, this effect was limited to ISOIs and durations that produced little or no motion reports. In addition, the three-way interaction accounted for only $2 \%$ of the total variance, while the duration $\times$ separation interaction accounted for $7 \%$ of the variance. This is consis- tent with the results of Strybel et al. (1990), who determined that separation had no effect on the ISOIs that produced auditory apparent motion for separations between $6^{\circ}$ and $160^{\circ}$. In the present investigation, we extended this separation down to $2.5^{\circ}$. For separations between $2.5^{\circ}$ and $160^{\circ}$, apparent motion was heard at the same range of ISOIs. Furthermore, the interaction of duration and ISOI does not appear to have been affected by separation, because the effect of duration obtained in the present experiment is similar to the effect reported by Briggs and Perrott (1972) at similar durations. Briggs and Perrot used a dichotic listening task, with a separation of $180^{\circ}$.

\section{Relationship to Visual Apparent Motion}

This minimum duration requirement represents another difference between the auditory and visual illusions of apparent motion. Although a test of the minimum duration for visual apparent motion has not been reported, the visual illusion has been observed with durations below $20 \mathrm{msec}$. Neuhaus (1930) obtained reports of apparent motion for durations as low as $10 \mathrm{msec}$. More recently, Baker and Braddick (1985) obtained motion reports for durations of $5 \mathrm{msec}$. Therefore, the illusion of visual apparent motion can be seen at durations that do not produce the perception of auditory apparent motion.

On the other hand, the visual illusion is affected by the spatial separation between the stimuli, whereas the auditory illusion is not. Because the auditory system is primarily a temporal analyzer, and because the visual system is primarily a spatial analyzer, it seems that the illusion in each modality is affected most by the factor to which it is most sensitive.

\section{Relationship to Auditory Motion Perception}

In vision, the illusion of apparent motion is used to investigate the processes involved in the perception of real visual motion. If the same assumption is valid in the auditory modality, our results could provide information on the processes involved in the perception of real motion in the auditory modality. In the present experiment, the optimal condition for continuous motion was produced by a 50-msec noise burst emitted in one location, followed immediately by a second 50 -msec burst in a second location (ISOI $=50 \mathrm{msec}$ ). Thus, the optimal stimulus sequence consisted of a "moving" event of $100 \mathrm{msec}$ with no silent interval. When these conditions are not met, motion is rarely heard.

For a stimulus duration of $10 \mathrm{msec}$, a stimulus sequence lasting $100 \mathrm{msec}$ is possible only when a relatively long silent period is placed between the leading and lagging stimuli (ISOI $=90 \mathrm{msec}$ ). Continuous motion was rarely reported in this condition, however. At a 10-msec duration, the optimal ISOI for continuous motion was $15 \mathrm{msec}$, which is a stimulus sequence with a silent interval of $5 \mathrm{msec}$ between the lead and lag stimuli. This might represent a compromise between the requirements for motion, and as a result, motion is rarely heard. 
The conclusion regarding stimulus duration is compatible with the results of Grantham (1986), who used a "simulated" motion paradigm to measure the minimum audible movement angle or the minimum travel required to detect the direction of a moving acoustic event. Grantham concluded that a minimum integration time of $150-300 \mathrm{msec}$ is required for subjects to detect motion. Our finding of a minimum duration for the apparently moving event is compatible with this observation, although our estimate of the time period $(100 \mathrm{msec})$ is slightly lower. This difference could be the result of the different stimuli used ("simulated" vs. "apparent" motion) or the result of the different tasks involved. In Grantham's experiment, subjects were presented with two stimuli, one "moving" and one stationary, and the subject was instructed to indicate which was the dynamic event.

In summary, we have determined that the perception of apparent motion is affected by the duration of the stimuli. To date, stimulus duration is the only factor that interferes with the perception of motion when the ISOI is optimal for the illusion. It appears that a minimum duration of 10-50 msec is required for the illusion to be heard. Although this value is dependent to some extent on the particular listener, it is not affected by the separation between the sources over the range tested here. This represents another difference between the auditory and visual illusions of apparent motion.

\section{REFERENCES}

ANStis, S. M. (1978). Apparent movement. In R. Held, W. Leibowitz, \& H. Teuber (Eds.), Handbook of sensory physiology: Vol. 8. Perception (pp. 655-673). Berlin: Springer-Verlag.

BAKER, C. L., BRAdDick, O. J. (1985). Temporal properties of the short-range process in apparent motion. Perception, 14, 181-192.

Briggs, R., \& Perrott, D. R. (1972). Auditory apparent movement under dichotic listening conditions. Joumal of Experimental Psychology, 92, 83-91.

GRANTHAM, D. W. (1986). Detection and discrimination of simulated motion of auditory targets in the horizontal plane. Jourmal of the Acoustical Society of America, 79, 1939-1949.

Kolers, P. A. (1972). Aspects of motion perception. Oxford: Pergamon. KORTE, A. (1915). Kinematoskopische Untersuchungen. Zeitschrifi fur Psychologie, 72, 193-206.

Neuhaus, W. (1930). Experimentelle Untersuchungen der Scheinbewegung. Pflugers Archiv fir die Gesamre Psychologie des Menschen und der Tiere, 75, 415-458.

Perrott, D. R. (1974). Auditory apparent motion. Journal of Auditory Research, 14, 163-169.

Strybel, T. Z., Manugas, C. L., Chan, O., Perrott, D. R. (1990). A comparison of the effects of spatial separation on apparent motion in the auditory and visual modalities. Perception \& Psycholophysics, 47, 439-448.

Strybel, T. Z., Manligas, C. L., \& Perrott, D. R. (1989). Auditory apparent motion under binaural and monaural listening conditions. Perception \& Psychophysics, 45, 371-377.

(Manuscript received June 20, 1991; revision accepted for publication January 22, 1992.) 